Título: Influencia de la presión arterial al inicio de las descompensaciones en el pronóstico de pacientes con insuficiencia cardiaca.

Title: Influence of blood pressure at the beginning of decompensation in prognosis of patients with heart failure.

Nombres y afiliaciones de los autores: Silvia Torrente Iranzo ${ }^{1}$, Vanesa Garcés Horna ${ }^{2,3}$, Claudia Josa Laorden ${ }^{2,3}$, Jorge Rubio Gracia ${ }^{2,3}$, Fernando José Ruiz Laiglesia ${ }^{2,3}$, Marta Sánchez Marteles ${ }^{2,3}$, Juan Ignacio Pérez Calvo ${ }^{1,2,3}$.

Autor para correspondencia: Silvia Torrente Iranzo. siltorrenteir@gmail.com

\footnotetext{
${ }^{1}$ Facultad de Medicina. Universidad de Zaragoza. Avda. Domingo Miral s/n, 50009. Zaragoza, España.

${ }^{2}$ Servicio de Medicina Interna, Hospital Clínico Universitario “Lozano Blesa”, Zaragoza. Avda. San Juan Bosco, no 15, 50009. Zaragoza, España.

${ }^{3}$ Instituto de Investigación Sanitaria de Aragón. Avda. San Juan Bosco no 9, 50009. Zaragoza, España.
} 


\section{INFLUENCIA DE LA PRESIÓN ARTERIAL EN EL INICIO DE LAS DESCOMPENSACIONES EN EL PRONÓSTICO DE PACIENTES CON INSUFICIENCIA CARDIACA.}

\section{RESUMEN}

Objetivo: existe una relación inversa entre las cifras de presión arterial (PA) en las descompensaciones y el pronóstico de la insuficiencia cardiaca (IC). Las características de esta relación no son bien conocidas. El objetivo del estudio fue analizar si esta relación se mantiene en una cohorte no seleccionada de pacientes con IC y si el tratamiento la modifica.

Material y métodos: estudio prospectivo de cohortes de pacientes ingresados por IC descompensada en un servicio de Medicina Interna y seguidos ambulatoriamente en una consulta monográfica. Los pacientes fueron agrupados en función de presión arterial sistólica (PAS) y diastólica (PAD); se analizaron las características clínicas, mortalidad global y reingresos al 1으, 3으 y 6 o mes de seguimiento.

Resultados: se incluyeron 221 pacientes tras un ingreso índice por IC. Media de edad 79,5 años (DE 8,09); varones 115. No hubo diferencias significativas en las características basales de los pacientes en función de los cuartiles de PAS. Los pacientes con menor PAS (Q1) tenían mayor mortalidad $(20 \%, p<0,05)$. No se encontraron diferencias para la PAD. Sin embargo, el análisis de Kaplan-Meier mostró mayor mortalidad global en los pacientes con menor PAS y PAD (log Rank= 0,011 y 0,041 respectivamente). Las características del tratamiento farmacológico no diferían entre los grupos del estudio.

Conclusión: en pacientes con IC no seleccionados, las cifras elevadas de PAS al ingreso se asocian con una menor mortalidad durante el seguimiento. El tratamiento farmacológico de la IC no parece influir en la relación inversa entre PAS al ingreso y la mortalidad.

Palabras clave: presión arterial, insuficiencia cardiaca, epidemiología reversa. 


\section{INFLUENCE OF BLOOD PRESSURE AT THE BEGINNING OF DECOMPENSATION IN PROGNOSIS OF PATIENTS WITH HEART FAILURE}

\section{ABSTRACT}

Objectives: A reverse relationship has been described between blood pressure (BP) and prognosis in acute heart failure (HF). The characteristics of this relationship are unknown. The objective of this study was to determine if this relationship is shown in a cohort of non-selected patients of a monographic consultation of HF and if it can be modified by treatment.

Materials and methods: cohorts prospective study including patients hospitalized for acute decompensated heart failure in Internal Medicine and followed as outpatients in a monographic consultation. The patients were classified according to their levels of systolic blood pressure (SBP) and diastolic blood pressure (DBP). Clinical characteristics, all-cause mortality and readmissions after the $1^{\text {st }}, 3^{\text {rd }}$ and $6^{\text {th }}$ month of follow-up were analyzed.

Results: 221 patients were included after their admission for acute HF. 79.5 years-old on average (ED 8,09); 115 male. Significant differences between SBP quartiles and basal characteristics were not found. Patients with lower SBP (Q1) had higher mortality (20\%, $p<0,05)$. Significant differences between mortality/readmissions and DBP were not found. However, the Kaplan- Meier analysis showed higher all cause mortality for the group of patients with lower SBP and DBP (log rank=0,011 and 0,041 respectively). There were no significant differences of treatment between each group of the study.

Conclusions: For non-selected patients suffering of acute decompensated HF, higher SBP during the admission is associated with significantly lower all-cause mortality during follow-up.

Pharmacologic treatment does not seem to influence this inverse relationship between SBP at admission and mortality.

Key words: blood pressure, heart failure, reverse epidemiology. 


\section{INTRODUCCIÓN}

La insuficiencia cardiaca (IC) es la principal causa de ingreso en los servicios de Medicina Interna (SMI) y conlleva una alta morbimortalidad. La prevalencia de la IC en Europa es de un 1-2\% de la población y sus tasas de supervivencia son menores que las del cáncer de mama, intestino o próstata ${ }^{1}$.

El sobrepeso y la hipertensión arterial (HTA) son factores de riesgo conocidos para el desarrollo de enfermedades cardiovasculares, entre otras la IC. No obstante, estudios recientes sugieren que una vez establecida la IC, ambos parecen comportarse como factores predictores de mejor pronóstico, asociados a una menor mortalidad durante el seguimiento. A estos fenómenos, todavía mal caracterizados, se les ha denominado "paradoja de la hipertensión y la obesidad", respectivamente y se engloban en el concepto un tanto genérico de epidemiología reversa ${ }^{2,3}$. EI tratamiento farmacológico de la IC con fracción de eyección reducida (ICFEr) ha demostrado su eficacia en la reducción de la morbimortalidad ${ }^{4}$. En la IC con fracción de eyección preservada (ICFEp) no existe ningún tratamiento que haya demostrado un claro beneficio, pero es habitual que se utilicen los mismos fármacos que en la ICFEr y la mortalidad de ambas es similar o discretamente superior en la ICFEr ${ }^{5,6}$. Es posible que la relación inversa observada entre PA tomada al inicio de las descompensaciones y el pronóstico de la IC pueda modificarse durante el seguimiento por la optimización del tratamiento farmacológico de acuerdo a las guías de práctica clínica (GPC) ${ }^{7}$.

El objetivo principal de este estudio fue analizar si existía relación entre la presión arterial sistólica (PAS), diastólica (PAD) y los reingresos o la mortalidad en el seguimiento de pacientes previamente ingresados por IC descompensada, seguidos en una consulta monográfica, y si esta relación se mantiene independientemente del tratamiento administrado.

\section{PACIENTES Y MÉTODOS}

Se trata de un estudio observacional prospectivo de cohortes.

Se incluyeron todos aquellos pacientes dados de alta de modo consecutivo tras un episodio de hospitalización por IC, entre junio de 2010 y octubre de 2014 y que fueran a ser seguidos en la consulta monográfica de IC. Se realizó una visita reglada al 1ㅇ, 3ㅇ y 60 mes. El criterio de derivación fue clínico, quedando a criterio del facultativo responsable, sin restricciones al respecto. La decisión de remitir al paciente a la consulta monográfica se siguió por criterios clínicos y de biomarcadores, seleccionando a aquellos pacientes que "a priori" podían tener peor pronóstico y por tanto beneficiarse de un tratamiento más ajustado. Los pacientes dieron su consentimiento por escrito para la obtención de los datos y su uso con fines de investigación para el estudio.

Los criterios de inclusión fueron: pacientes mayores de 18 años con el diagnóstico clínico de IC aguda (de "novo" o IC crónica agudizada) y concentraciones de fragmento aminoterminal del 
péptido natriurético cerebral (NT-proBNP) $>1000 \mathrm{pg} / \mathrm{mL}$ al ingreso. Se excluyeron aquellos con deterioro cognitivo, esperanza de vida menor a 6 meses, neoplasia activa, insuficiencia renal avanzada (filtrado glomerular $<30 \mathrm{ml} / \mathrm{min} / 1,73 \mathrm{~m}^{2}$ según la fórmula Modification of Diet of Renal Disease [MDRD-4]), tratamiento inotrópico al ingreso o intolerancia o alergia al tratamiento diurético.

Tras el ingreso índice, todos los pacientes mantuvieron un seguimiento ambulatorio en la consulta de IC, al menos al mes, tres y seis meses del alta.

Para analizar la relación entre la PA y los eventos en el seguimiento, se tomó como referencia la primera toma de TA registrada a la llegada del paciente a la sala de Urgencias del hospital.

Se consideraron eventos primarios la mortalidad por IC y la variable combinada de mortalidad y reingresos por IC a los 180 días. También se analizó la mortalidad global. Se recogieron los datos demográficos, analíticos y ecográficos mediante la revisión de las historias clínicas. En las primeras 24-48 horas del ingreso hospitalario se determinó la función renal mediante las concentraciones de urea, creatinina y cistatina séricas; se midieron los iones, hemoglobina, hematocrito, proteínas totales, albúmina, colesterol total y NT-proBNP.

Se consideró ICFEp a aquellos pacientes con una FEVI $\geq 50 \%$.

Análisis estadístico. Se empleó el programa SPSS (Statistical Package for the Social Sciences) 15.0 (SPSS Inc. Chicago, EEUU). Tras un análisis descriptivo de la muestra, la dividimos en cuatro grupos con igual número de pacientes según la PAS y PAD, utilizando Chi cuadrado para variables categóricas y análisis de la varianza (ANOVA) para variables cuantitativas. Analizamos el porcentaje de reingresos y fallecimientos al 1으, 3으 y 6으 mes, y su significación estadística. También se realizaron curvas de supervivencia de Kaplan-Meier. Se consideraron significativas las diferencias con un valor de $p<0,05$.

\section{RESULTADOS}

Se incluyeron 221 pacientes (115 varones y 106 mujeres), con una media de edad de 79,51 años (DE 8,09). En la Tabla 1 se muestran las características basales de los pacientes. La media de la FEVI fue de 52,80\%; un 56\% de los pacientes tenían una FEVI $\geq 50 \%$. En la Tabla 1 se recoge el tratamiento de los pacientes al alta.

En las Tablas 2 y 3 se muestran las características clínicas y analíticas de los pacientes distribuidos según los cuartiles de PAS y PAD respectivamente. Los pacientes en el Q4 de PAS (PAS> 157,5mmHg) tenían una PAD significativamente más alta, y menor frecuencia de pacientes cuya causa primaria de IC era la valvulopatía aórtica. Respecto a la PAD, los pacientes en el Q4 (PAD > 81 $\mathrm{mmHg}$ ) tenían una PAS significativamente más alta, eran más jóvenes y tenían unas concentraciones de hemoglobina significativamente más elevadas. Además, en 
dichos pacientes la proporción de los inhibidores de la enzima conversora de la angiotensina (IECA) era mayor.

Se realizó un análisis similar para el IAMC de los pacientes, no encontrando diferencias significativas en cuanto a características basales, tratamiento recibido o pronóstico.

En la Tabla 4 se muestran el análisis de los reingresos y mortalidad global según los cuartiles de PAS y PAD. La mortalidad fue significativamente menor en los pacientes de los cuartiles con mayor PAS (Q1 20\%; Q2 9,1\%; Q3/Q4 5,5\%; p=0,034). Respecto a los reingresos no hubo diferencias significativas entre los diferentes cuartiles de PAS, aunque sí que se observa una tendencia a un mayor porcentaje de reingresos en el cuartil de menor PAS.

La mortalidad global y los reingresos en función de los cuartiles de PAD siguen la misma tendencia que para los cuartiles de PAS aunque no llegan a alcanzar significación estadística. La curva de supervivencia (Figuras 1 y 2), mostró una relación inversa y significativa entre los cuartiles de PAS y PAD y la mortalidad (Log Rank test 0,011). Al incluir el tiempo de seguimiento, también la supervivencia mostró una relación inversa y significativa entre mortalidad y PAD (log Rank test 0,041) que no se encuentra cuando se analizan las diferencias brutas entre cuartiles.

Figura 1. Curva de supervivencia según Presión Arterial Sistólica.

Figura 2. Curva de supervivencia según Presión Arterial Diastólica.

\section{DISCUSIÓN}

Nuestros resultados muestran que, en pacientes ingresados por con IC existe una relación entre la PAS medida al inicio de la descompensación y el pronóstico, de modo que aquellos con PAS más alta tienen un mejor pronóstico tras el alta, en términos de mortalidad por cualquier causa.

En nuestra serie son de destacar dos aspectos. En primer lugar, el corto periodo de seguimiento, 6 meses, que probablemente denota la intensidad de la asociación entre la PAS y una mayor supervivencia. En segundo lugar, al tratarse de una población seguida en una consulta monográfica, el tratamiento está optimizado, al menos considerando la edad y comorbilidades de los pacientes, y no difiere entre los pacientes de los distintos cuartiles de PA, salvo respecto a la toma de IECA, que es más frecuente en los pacientes con cifras de PA más elevadas. A pesar de ello, es difícil que este hecho por si solo justifique las diferencias en la mortalidad halladas ya que se trata de una cohorte de pacientes con una alta proporción de pacientes con ICFEp en la que el efecto beneficioso de los diferentes grupos farmacológicos no ha demostrado un beneficio claro en la mortalidad. Este hecho puede indicar sugiere que 
la relación entre PAS y pronóstico es independiente del tratamiento administrado, enfatizando de nuevo lo robusto de esta asociación.

El fenómeno denominado "epidemiología reversa" es un término descriptivo. Pretende llamar la atención sobre una situación aparentemente paradójica. Un factor de riesgo de IC como la hipertensión arterial, por tanto anterior a la misma, que incluso es precipitante de las descompensaciones, se asocia con un mejor pronóstico una vez que el daño en el órgano diana se hace expresivo clínicamente. Se desconoce la fisiopatología de esta asociación, así como si se trata de un fenómeno específico de la IC. De hecho, en otros trastornos crónicos como la EPOC no se ha descrito un fenómeno similar e incluso en la insuficiencia renal crónica el pronóstico mejora paralelamente a la reducción de la $\mathrm{PA}^{8}$.

Algunos autores proponen que esta asociación inversa constituye un fenotipo distinto de IC 2,9. Por el contrario, otros suponen que la asociación inversa entre PA elevada y pronóstico es un mero marcador de la reserva funcional del corazón. En este sentido, los pacientes con IC y elevada PA en las agudizaciones tendrían una mejor función de contráctil y una perfusión tisular menos deteriorada, por lo que su organismo respondería mejor frente a situaciones de sobrecarga hemodinámica ${ }^{10}$. Es plausible también que un mayor tono simpático y una PA más elevada ocasionen síntomas de IC más precoces, con lo que el diagnóstico de IC se realizaría de forma temprana administrándose un tratamiento más precoz de las agudizaciones que reduciría el riesgo de complicaciones.

Algunos autores proponen que el fenómeno de la epidemiología reversa sólo-se cumple en determinados grupos de pacientes. Se debate si la mejoría pronóstica y las cifras más elevadas de PA al inicio de una descompensación es un fenómeno que afecta por igual a todos los pacientes con IC o es propio de algún subgrupo, dependiente de la etiología u otros factores. En este sentido, los primeros estudios se realizaron en pacientes con FEVI reducida ${ }^{11}$. Sin embargo, en nuestro estudio, con una alta proporción de pacientes con FEVI preservada, la asociación se mantiene, algo que han encontrado también otros autores ${ }^{12}$. Nuestros resultados proceden de una muestra limitada de pacientes, por lo que es difícil extraer conclusiones más allá de la mera descripción, más aún acerca de la posible causalidad de la relación. Sin embargo, la alta proporción de pacientes con ICFEp y el espectro de comorbilidades, son sugestivos de que la relación entre elevación de la PA y el pronóstico en la IC es un fenómeno inespecífico no vinculado a una etiología concreta.

Un estudio reciente aporta una idea hasta ahora poco investigada. Güder et a ${ }^{13}$ realizaron un análisis incluyendo 3315 pacientes clasificados según estadio de IC, y sugieren que la asociación inversa entre una mayor PA y el mejor pronóstico de los pacientes con IC ocurre sólo en los estadios sintomáticos de la IC. 
También se ha debatido si es mejor marcador pronóstico la PAS, PAD o PA media (PAM)2 ${ }^{2}$, en nuestro estudio encontramos claramente una asociación con la PAS, coincidiendo con otros estudios previos ${ }^{12,13}$.

La relación observada entre PAS y mortalidad global sigue un modelo lineal, al contrario de lo que se afirma en otros estudios sobre una posible relación en forma de " $U$ " y una relación lineal sólo en los casos de FEVI inferior al 30\% ${ }^{12}$. Hay que tener en cuenta que en nuestra muestra hay pocos pacientes con cifras extremas de PAS $(<110 \mathrm{mmHg},>160 \mathrm{mmHg})$ y por tanto no podemos descartar la posibilidad de una relación en forma de " $U$ " entre mortalidad y los distintos cuartiles.

La ausencia de relación de los reingresos con respecto a los cuartiles de PAS y PAD, podría deberse a que los pacientes de nuestra serie están incluidos en visitas de seguimiento en una consulta especializada y por tanto tienen mayor adherencia al tratamiento y revisiones periódicas programadas que disminuye la frecuentación de urgencias y los reingresos ${ }^{14,15,16}$. Además, los reingresos son un "end point" discutible, ya que no todos los hospitales tienen los mismos criterios de ingreso ni recursos y, por supuesto, la influencia del médico de Atención Primaria (MAP) puede haber sido decisiva en la resolución de episodios sin necesidad de reingreso ${ }^{16,17}$.

Limitaciones destudio: se trata de una muestra de pacientes pequeña y con un periodo de seguimiento limitado a 6 meses. Por otro lado, los pacientes fueron seleccionados durante su ingreso, con un criterio clínico, priorizando aquellos que por sus características clínicas y de biomarcadores se podría presuponer un peor pronóstico. Este criterio supone un sesgo de selección al excluir pacientes con comorbilidades neurológicas o situaciones de aislamiento que impiden o dificultan su traslado a las consultas. No obstante la muestra de pacientes creemos que es representativa de la práctica clínica real. Además empleamos la mortalidad global, y no la mortalidad por causa cardiaca como objetivo, lo que podría incluir sesgos de selección a favor de los pacientes con FEVI preservada, mayoritarios en la muestra.

Por otra parte la limitación del tamaño muestral nos impide analizar el comportamiento de los cuartiles de PA en función de la FEVI.

Se necesitan nuevos y más amplios estudios para comprender el fenómeno de la epidemiología reversa. Sise demuestra que este fenómenoes algo-sistemático una vez establecida la IC, deberíamos preguntarnos silos factores de riesgo cardiovascular clásicos deben ser tratados en los pacientes con $1 \mathrm{C}$ del mismo modo y con la misma agresividad que en la población general.

En conclusión, en nuestro estudio una PAS elevada al ingreso de una descompensación por IC se asocia de modo independiente a un mejor pronóstico en términos de mortalidad por cualquier causa tras el alta. La relación es lineal e inversa, se muestra ya al tercer mes y se 
mantiene durante los 6 meses de seguimiento. Esta asociación no parece influirse por el tratamiento de la IC.

\footnotetext{
${ }^{1}$ Ponikowski P, Anker SD, AlHabib KF, Cowie MR, Force TL, Hu S, et al. Heart failure: preventing disease and death worldwide. ESC Heart Failure 2014;1:4-25.
}

${ }^{2}$ Pérez-Calvo Jl, Montero-Pérez-Barquero M, Camafort-Babkowski M, Conthe-Gutiérrez P, Formiga F, AramburuBodas $\mathrm{O}$, et al. Influence of admission blood pressure on mortality in patients with acute decompensated heart failure. QJM 2011;104:325-33.

${ }^{3}$ Trullàs JC, Formiga F, Montero M, Conde A, Casado J, Carrasco FJ, et al. Paradoja de la obesidad en la insuficiencia cardiaca. Resultados del registo RICA. Med Clin (Barc) 2011;137:671-677.

${ }^{4}$ McMurray JJ. Improving outcomes in heart failure: a personal perspective. Eur Heart J 2015;36:3467-70.

${ }^{5}$ Bytyçi I, Bajraktari G. Mortality in heart failure patients. Anatolian J Cardiol 2015;15:63-8.

${ }^{6}$ Meta-analysis Global Group in Chronic Heart Failure (MAGGIC). The survival of patients with heart failure with preserved or reduced left ventricular ejection fraction: an individual patient data meta-analysis. Eur Heart J 2012;33:1750-7.

${ }^{7}$ Ponikowski P, Voors AA, Anker SD, Bueno H, Cleland JG, Coats AJ, et al. 2016 ESC Guidelines for the diagnosis and treatment of acute and chronic heart failure: The Task Force for the diagnosis and treatment of acute and chronic heart failure of the European Society of Cardiology (ESC). Developed with the special contribution of the Heart Failure Association (HFA) of the ESC. Eur J Heart Fail 2016;18:891-975.

${ }^{8}$ Lv J, Ehteshami P, Sarnak MJ, Tighiouart H, Jun M, Ninomiya T, et al. Effects of intensive blood pressure lowering on the progression of chronic kidney disease: a systematic review and meta-analysis. CMAJ 2013;185:949-57.

${ }^{9}$ Milo-Cotter O, Adams KF, O'Connor CM, Uriel N, Kalusi E,Felker GM, et al. Acute heart failure associated with high admission blood pressure- A distinct vascular disorder? Eur J Heart Fail 2007;9:178-83.

${ }^{10}$ Güder G, Frantz S, Bauersachs J, Allolio B, Wanner C, Koller MT, et al. Reverse epidemiology in systolic and nonsystolic heart failure: cumulative prognostic benefit of classical cardiovascular risk factors. Circ Heart Fail 2009;2:563-71.

${ }^{11}$ Ather S, Chan W, Chillar A, Aguilar D, Pritchett AM, Ramasubbu K, et al. Association of systolic blood pressure with mortality in patients with heart failure with reduced ejection fraction: a complex relationship. Am Heart J 2011;161:567-73.

${ }^{12}$ Buiciuc O, Rusinaru D, Lévy F, Peltier M, Slama M, Leborgne L, et al. Low systolic blood pressure at admission predicts long-term mortality in heart failure with preserved ejection fraction. J Card Fail 2011;17:907-15.

${ }^{13}$ Güder G, Gelbrich G, Edelmann F, Wachter R, Pieske B, Pankuweit S, et al. Reverse epidemiology in different stages of heart failure. Int J Cardiol 2015;184:216-24.

${ }^{14}$ Ducharme A, Doyon O, White M, Rouleau JL, Brophy JM. Impact of care at a multidisciplinary congestive heart failure clinic: a randomized trial. CMAJ 2005;173:40-5.

${ }^{15}$ Feltner C, Jones CD, Cené CW, Zheng ZJ, Sueta CA, Coker-Schwimmer EJ, et al. Transitional care interventions to prevent readmissions for persons with heart failure: a systematic review and meta-analysis. Ann Intern Med 2014;160:774-84. 
${ }^{16}$ Cerqueiro JM, González-Franco A, Montero-Pére<-Barquero M, Llácer P, Conde A, Dávila MF. Reducción de ingresos y visitas a Urgencias en pacientes frágiles con insuficiencia cardíaca: resultados del programa asistencial UMIPIC. Rev Clin Esp 2016;216:22-5.

${ }^{17}$ Kasper EK, Gerstenblith G, Hefter G, Van Anden E, Brinker JA, Thiemann DR, et al. A randomized trial of the efficacy of multidisciplinary care in heart failure outpatients at high risk of hospital readmission. J Am Coll Cardiol 2002;39:471-80. 
Tabla 1. Características de los pacientes

\begin{tabular}{|c|c|}
\hline & Media (desv típica) \\
\hline Edad (años) & $79,51(8,09)$ \\
\hline IMC (kg/m2) & $29,32(5,63)$ \\
\hline Frecuencia cardiaca (Ipm) & $86,47(22,56)$ \\
\hline PAS (mmHg) & $138,92(29,46)$ \\
\hline PAD (mmHg) & $73,57(14,67)$ \\
\hline FEVI (\%) & $52,80(13,97)$ \\
\hline NT-proBNP (pg/ml) & $5339,58(6179,22)$ \\
\hline Hemoglobina (g(dl) & $12,2(2,09)$ \\
\hline Creatinina (mg/dl) & $1,25(0,69)$ \\
\hline \multirow[t]{2}{*}{ Sodio (mEq/H) } & $141,87(3,91)$ \\
\hline & $\begin{array}{c}\text { Número } \\
\text { (porcentaje) }\end{array}$ \\
\hline Hombres & 115 (52\%) \\
\hline Mujeres & $106(48 \%)$ \\
\hline Fumadores & $26(11,8 \%)$ \\
\hline \multicolumn{2}{|l|}{ Causa primaria de la IC } \\
\hline Cardiopatia isquémica & $50(22,6 \%)$ \\
\hline HTA & $93(42,1 \%)$ \\
\hline Valvulopatía mitral & $22(10 \%)$ \\
\hline Valvulopatía aórtica & $20(9 \%)$ \\
\hline Valvulopatía derecha & $1(0,5 \%)$ \\
\hline EPOC & $8(3,6 \%)$ \\
\hline Alcoholisme & $3(1,4 \%)$ \\
\hline Enfermedad pulmonar no & $z(0,9 \%)$ \\
\hline EPOC & \\
\hline Otros & $21(9,5 \%)$ \\
\hline \multicolumn{2}{|l|}{ NYHA basal } \\
\hline 1 & $23(10,4 \%)$ \\
\hline II & $139(62,9 \%)$ \\
\hline III & $57(25,8 \%)$ \\
\hline IV & $1(0,5 \%)$ \\
\hline \multicolumn{2}{|l|}{ Enfermedades asociadas } \\
\hline Isquemia cardiaca & $57(25,8 \%)$ \\
\hline EPOC & $65(29,4 \%)$ \\
\hline HTA & $163(73,8 \%)$ \\
\hline Fibrilación auricular & $140(63,3 \%)$ \\
\hline Diabetes mellitus & $75(33,9 \%)$ \\
\hline Insuficiencia renal & $76(34,4 \%)$ \\
\hline Anemia & $70(31,7 \%)$ \\
\hline Tratamientos & $\begin{array}{c}\text { Número } \\
\text { (porcentaje) }\end{array}$ \\
\hline Diuréticos asa & $172(77,8 \%)$ \\
\hline
\end{tabular}




\begin{tabular}{|c|c|}
\hline Diuréticos Tiazídicos & $24(10,9 \%)$ \\
\hline Antialdosterónico & $57(25,8 \%)$ \\
\hline Digoxina & $50(22,6 \%)$ \\
\hline IECAs & $94(42,5 \%)$ \\
\hline ARA II & $65(29,4 \%)$ \\
\hline Beta Bloqueante & $128(57,9 \%)$ \\
\hline Anticoagulante & $124(56,1 \%)$ \\
\hline Antiagregante & $56(25,3)$ \\
\hline \multicolumn{2}{|l|}{ Antidiabéticos } \\
\hline ADOs & $32(14,5 \%)$ \\
\hline Insulina & $26(11,8 \%)$ \\
\hline ADOs+ Insulina & $4(1,8 \%)$ \\
\hline \multicolumn{2}{|l|}{ Hierro/EPO } \\
\hline Hierro & $40(18,1 \%)$ \\
\hline EPO & $6(2,7 \%)$ \\
\hline EPO + Hierro & $2(0,9 \%)$ \\
\hline
\end{tabular}

ADOS: antidiabéticos orales; ARA II: Antagonistas de los Receptores de la Angiotensina II; EPO: Eritropoyetina; EPOC: Enfermedad Pulmonar Obstructiva crónica; FEVI: Fracción de Eyección del Ventrículo Izquierdo; HTA: Hipertensión Arterial; IECAs: Inhibidores de la Enzima Conversora de la Angiotensina; IMC: Índice de Masa Corporal; NT-proBNP: fracción aminoterminal del péptido natriurético cerebral; NYHA: New York Heart Association; PAD: Presión Arterial Diastólica; PAS: Presión Arterial Sistólica. 
Tabla 2. Características clínicas y analíticas de los pacientes según Presión Arterial Sistólica.

\begin{tabular}{|c|c|c|c|c|c|}
\hline & Q1 $(<119,25)$ & $\begin{array}{c}\text { Q2 }(119,25- \\
137,5)\end{array}$ & $\begin{array}{c}\text { Q3 }(137,5- \\
157,75)\end{array}$ & Q4 $(>157,5)$ & $p$ \\
\hline & $n=55$ & $n=55$ & $n=55$ & $n=55$ & \\
\hline Edad (años) & $79,85(6,97)$ & $78,87(9,38)$ & $80,85(8,07)$ & $77,94(7,69)$ & 0,270 \\
\hline IMC (kg/m2) & $28,99(4,70)$ & $29,31(5,26)$ & $29,74(5,93)$ & $29,33(6,64)$ & 0,932 \\
\hline $\begin{array}{l}\text { Frecuencia cardiaca } \\
\text { (Ipm) }\end{array}$ & $88,22(28,82)$ & $88,02(23,01)$ & $84,47(20,15)$ & $85,49(17,08)$ & 0,776 \\
\hline PAS (mmHg) & $103,44(11,44)$ & $127,95(5,27)$ & $146,27(5,21)$ & $178,04(17,75)$ & 0,000 \\
\hline PAD (mmHg) & $61,56(11,56)$ & $70,85(10,81)$ & $76,35(12,68)$ & $85,53(12,41)$ & 0,000 \\
\hline FEVI (\%) & $50,73(16,00)$ & $53,06(12,88)$ & $52,52(12,61)$ & $54,94(14,16)$ & 0,493 \\
\hline NT-proBNP (pg/ml) & $\begin{array}{c}5573,90 \\
(4383,98)\end{array}$ & $\begin{array}{c}5444,43 \\
(6131,70)\end{array}$ & $\begin{array}{c}4637,15 \\
(5778,83)\end{array}$ & $\begin{array}{c}5815,12 \\
(8377,00)\end{array}$ & 0,812 \\
\hline Hemoglobina (g/dl) & $12,23(1,90)$ & $12,09(1,67)$ & $11,73(2,39)$ & $12,78(2,24)$ & 0,072 \\
\hline Creatinina (mg/dl) & $1,15(0,45)$ & $1,26(0,50)$ & $1,38(1,03)$ & $1,22(0,63)$ & 0,399 \\
\hline Sodio $(\mathrm{mEq} / \mathrm{H})$ & $141,79(3,78)$ & $141,41(3,87)$ & $141,71(4,01)$ & $142,57(4,02)$ & 0,459 \\
\hline Hombres & $54,50 \%$ & $45,50 \%$ & $50,90 \%$ & $58,20 \%$ & 0,583 \\
\hline Mujeres & $45,50 \%$ & $54,50 \%$ & $49,10 \%$ & $41,80 \%$ & - \\
\hline Fumadores & $14,80 \%$ & $11,10 \%$ & $12,70 \%$ & $9,10 \%$ & 0,821 \\
\hline \multicolumn{6}{|l|}{ Causa primaria de la IC } \\
\hline Cardiopatía isquémica & $32,70 \%$ & $18,20 \%$ & $9,30 \%$ & $30,90 \%$ & 0,026 \\
\hline HTA & $26 \%$ & $38,20 \%$ & $63,00 \%$ & $41,80 \%$ & \\
\hline Valvulopatía mitral & $9,10 \%$ & $14,50 \%$ & $7,40 \%$ & $9,10 \%$ & - \\
\hline Valvulopatía aórtica & $18,20 \%$ & $7,30 \%$ & $3,70 \%$ & $7,30 \%$ & - \\
\hline Valvulopatía derecha & $1,80 \%$ & $0,00 \%$ & $0,00 \%$ & $0,00 \%$ & - \\
\hline EPOC & $3,60 \%$ & $3,60 \%$ & $3,70 \%$ & $3,60 \%$ & - \\
\hline Alcoholismo & $0,00 \%$ & $1,80 \%$ & $3,70 \%$ & $0,00 \%$ & - \\
\hline Enfermedad pulmonarno & $1,80 \%$ & $0,00 \%$ & $1,90 \%$ & $0,00 \%$ & - \\
\hline EPOC & & & & & \\
\hline Otros & $7,30 \%$ & $16,40 \%$ & $7,40 \%$ & $7,30 \%$ & - \\
\hline \multicolumn{6}{|l|}{ NYHA basal } \\
\hline 1 & $1,80 \%$ & $14,50 \%$ & $12,70 \%$ & $12,70 \%$ & 0,375 \\
\hline II & $65,50 \%$ & $63,60 \%$ & $63,60 \%$ & $60,00 \%$ & \\
\hline III & $30,90 \%$ & $20,00 \%$ & $23,60 \%$ & $27,30 \%$ & \\
\hline IV & $1,80 \%$ & $0,00 \%$ & $0,00 \%$ & $0,00 \%$ & \\
\hline \multicolumn{6}{|l|}{ Comorbilidades } \\
\hline Isquemia cardiaca & $29,10 \%$ & $27,30 \%$ & $18,20 \%$ & $29,10 \%$ & 0,504 \\
\hline EPOC & $21,80 \%$ & $37,00 \%$ & $32,70 \%$ & $27,30 \%$ & 0,330 \\
\hline HTA & $67,30 \%$ & $67,30 \%$ & $80,00 \%$ & $80,00 \%$ & 0,204 \\
\hline Fibrilación auricular & $65,50 \%$ & $60,00 \%$ & $72,70 \%$ & $56,40 \%$ & 0,306 \\
\hline Diabetes mellitus & $24,10 \%$ & $31,50 \%$ & $40,00 \%$ & $41,80 \%$ & 0,182 \\
\hline Insuficiencia renal & $31,50 \%$ & $43,60 \%$ & $29,60 \%$ & $34,50 \%$ & 0,425 \\
\hline Anemia & $32,70 \%$ & $25,50 \%$ & $41,80 \%$ & $27,30 \%$ & 0,250 \\
\hline
\end{tabular}




\begin{tabular}{|c|c|c|c|c|c|}
\hline Diuréticos asa & $93,80 \%$ & $83,30 \%$ & $88,20 \%$ & $93,20 \%$ & 0,309 \\
\hline Diuréticos Tiazídicos & $8,30 \%$ & $10,90 \%$ & $12,00 \%$ & $20,90 \%$ & 0,311 \\
\hline Antialdosterónico & $42,60 \%$ & $25,00 \%$ & $25,00 \%$ & $25,00 \%$ & 0,153 \\
\hline Digoxina & $27,10 \%$ & $20,80 \%$ & $36,50 \%$ & $18,20 \%$ & 0,165 \\
\hline IECAs & $38,30 \%$ & $45,70 \%$ & $67,30 \%$ & $50,00 \%$ & 0,032 \\
\hline ARA II & $25,00 \%$ & $40,40 \%$ & $31,40 \%$ & $39,50 \%$ & 0,341 \\
\hline Beta Bloqueante & $68,80 \%$ & $61,70 \%$ & $65,40 \%$ & $72,70 \%$ & 0,711 \\
\hline Anticoagulante & $64,60 \%$ & $67,40 \%$ & $74,50 \%$ & $55,80 \%$ & 0,294 \\
\hline Antiagregante & $36,20 \%$ & $29,80 \%$ & $23,50 \%$ & $30,20 \%$ & 0,599 \\
\hline \multicolumn{6}{|l|}{ Antidiabéticos } \\
\hline ADOs & $14,90 \%$ & $14,60 \%$ & $18,00 \%$ & $20,50 \%$ & 0,647 \\
\hline Insulina & $8,50 \%$ & $14,60 \%$ & $12,00 \%$ & $20,50 \%$ & \\
\hline ADOs + Insulina & $2,10 \%$ & $0,00 \%$ & $2,00 \%$ & $4,50 \%$ & \\
\hline \multicolumn{6}{|l|}{ Hierro/EPO } \\
\hline Hierro & $17,40 \%$ & $14,60 \%$ & $28,00 \%$ & $26,20 \%$ & 0,246 \\
\hline EPO & $2,20 \%$ & $2,10 \%$ & $8,00 \%$ & $0,00 \%$ & \\
\hline EPO + Hierro & $0,00 \%$ & $2,10 \%$ & $2,00 \%$ & $0,00 \%$ & \\
\hline
\end{tabular}

ADOS: antidiabéticos orales; ARA II: Antagonistas de los Receptores de la Angiotensina II; EPO: Eritropoyetina; EPOC: Enfermedad Pulmonar Obstructiva crónica; FEVI: Fracción de Eyección del Ventrículo Izquierdo; HTA: Hipertensión Arterial; IECAs: Inhibidores de la Enzima Conversora de la Angiotensina; IMC: Índice de Masa Corporal; NT-proBNP: fracción aminoterminal del péptido natriurético cerebral; NYHA: New York Heart Association; PAD: Presión Arterial Diastólica; PAS: Presión Arterial Sistólica.

Tabla 3. Características clínicas y analíticas de los pacientes según Presión Arterial Diastólica

Q1 (<64) $\quad$ Q2 (64-72) $\quad$ Q3 (72-81) $\quad$ Q4 (>81) $\quad$ p




\begin{tabular}{|c|c|c|c|c|c|}
\hline & $n=57$ & $n=56$ & $n=54$ & $n=53$ & \\
\hline Edad (años) & $81,25(5,62)$ & $78,32(8,14)$ & $80,55(7,86)$ & $77,36(9,91)$ & 0,037 \\
\hline IMC (kg/m2) & $29,51(4,41)$ & $29,29(6,25)$ & $29,20(5,42)$ & $29,32(6,51)$ & 0,993 \\
\hline $\begin{array}{l}\text { Frecuencia cardiaca } \\
\text { (Ipm) }\end{array}$ & $88,09(26,19)$ & $81,62(22,01)$ & $90,43(21,95)$ & $86,04(19,00)$ & 0,212 \\
\hline PAS (mmHg) & $114,33(19,69)$ & $\begin{array}{c}137,54 \\
(21,375)\end{array}$ & $144,96(29,86)$ & $160,86(25,70)$ & 0,000 \\
\hline PAD (mmHg) & $56,37(6,29)$ & $68,68(2,45)$ & $76,83(2,45)$ & $93,92(8,26)$ & 0,000 \\
\hline FEVI (\%) & $53,11(12,75)$ & $55,76(13,41)$ & $50,06(15,00)$ & $52,47(14,59)$ & 0,232 \\
\hline NT-proBNP (pg/ml) & $\begin{array}{c}5358,39 \\
(4983,77)\end{array}$ & $\begin{array}{c}4118,21 \\
(3310,32)\end{array}$ & $\begin{array}{c}5176,60 \\
(5795,01)\end{array}$ & $\begin{array}{c}6701,61 \\
(9329,15)\end{array}$ & 0,311 \\
\hline Hemoglobina (g/dl) & $11,88(2,05)$ & $11,35(2,18)$ & $12,28(1,83)$ & $13,40(1,77)$ & 0,000 \\
\hline Creatinina (mg/dl) & $1,20(0,50)$ & $1,40(1,04)$ & $1,17(0,41)$ & $1,23(0,63)$ & 0,294 \\
\hline Sodio (mEq/L) & $141,53(3,63)$ & $142,04(4,30)$ & $141,67(3,90)$ & $142,28(3,89)$ & 0,744 \\
\hline Hombres & $49,10 \%$ & $50,00 \%$ & $55,60 \%$ & $54,70 \%$ & 0,873 \\
\hline Mujeres & $50,90 \%$ & $50,00 \%$ & $44,40 \%$ & $45,30 \%$ & - \\
\hline Fumadores & $10,70 \%$ & $10,70 \%$ & $11,30 \%$ & $15,10 \%$ & 0,878 \\
\hline \multicolumn{6}{|l|}{ Causa primaria de la IC } \\
\hline Cardiopatía isquémica & $26,30 \%$ & $17,90 \%$ & $24,10 \%$ & $23,10 \%$ & 0,431 \\
\hline HTA & $31,60 \%$ & $42,90 \%$ & $50,00 \%$ & $44,20 \%$ & \\
\hline Valvulopatía mitral & $14,00 \%$ & $10,70 \%$ & $5,60 \%$ & $9,60 \%$ & - \\
\hline Valvulopatía aórtica & $15,80 \%$ & $8,90 \%$ & $7,40 \%$ & $3,80 \%$ & - \\
\hline Valvulopatía derecha & $1,80 \%$ & $0,00 \%$ & $0,00 \%$ & $0,00 \%$ & - \\
\hline EPOC & $0,00 \%$ & $5,40 \%$ & $1,90 \%$ & $7,70 \%$ & - \\
\hline Alcoholismo & $0,00 \%$ & $0,00 \%$ & $3,70 \%$ & $1,90 \%$ & - \\
\hline Enfermedad pulmonarno & $1,80 \%$ & $1,80 \%$ & $0,00 \%$ & $0,00 \%$ & - \\
\hline EPOC & & & & & \\
\hline Otros & $8,80 \%$ & $12,50 \%$ & $7,40 \%$ & $9,60 \%$ & - \\
\hline \multicolumn{6}{|l|}{ NYHA basal } \\
\hline 1 & $3,50 \%$ & $8,90 \%$ & $14,80 \%$ & $15,10 \%$ & 0,454 \\
\hline II & $64,90 \%$ & $67,90 \%$ & $61,10 \%$ & $58,50 \%$ & \\
\hline III & $29,80 \%$ & $21,40 \%$ & $24,10 \%$ & $26,40 \%$ & \\
\hline IV & $1,80 \%$ & $0,00 \%$ & $0,00 \%$ & $0,00 \%$ & \\
\hline \multicolumn{6}{|l|}{ Comorbilidades } \\
\hline Isquemia cardiaca & $26,30 \%$ & $30,40 \%$ & $24,10 \%$ & $22,60 \%$ & 0,808 \\
\hline EPOC & $30,40 \%$ & $33,90 \%$ & $29,60 \%$ & $24,50 \%$ & 0,760 \\
\hline HTA & $70,20 \%$ & $76,80 \%$ & $72,20 \%$ & $75,50 \%$ & 0,853 \\
\hline Fibrilación auricular & $71,90 \%$ & $57,10 \%$ & $66,70 \%$ & $58,50 \%$ & 0,316 \\
\hline Diabetes mellitus & $27,30 \%$ & $33,90 \%$ & $37,00 \%$ & $39,60 \%$ & 0,562 \\
\hline Insuficiencia renal & $37,50 \%$ & $35,70 \%$ & $34,00 \%$ & $32,10 \%$ & 0,942 \\
\hline Anemia & $36,80 \%$ & $42,90 \%$ & $25,90 \%$ & $20,80 \%$ & 0,054 \\
\hline \multicolumn{6}{|l|}{ Tratamiento recibido } \\
\hline Diuréticos asa & $95,70 \%$ & $89,60 \%$ & $86,50 \%$ & $86,40 \%$ & 0,407 \\
\hline Diuréticos Tiazídicos & $6,30 \%$ & $16,70 \%$ & $10,00 \%$ & $19,50 \%$ & 0,214 \\
\hline Antialdosterónico & $35,40 \%$ & $31,90 \%$ & $27,50 \%$ & $22,20 \%$ & 0,533 \\
\hline
\end{tabular}




\begin{tabular}{|c|c|c|c|c|c|}
\hline Digoxina & $27,10 \%$ & $29,20 \%$ & $21,60 \%$ & $26,70 \%$ & 0,847 \\
\hline IECAs & $29,50 \%$ & $48,90 \%$ & $60,00 \%$ & $62,20 \%$ & 0,007 \\
\hline ARA II & $34,80 \%$ & $37,50 \%$ & $30,00 \%$ & $33,30 \%$ & 0,887 \\
\hline Beta Bloqueante & $63,80 \%$ & $60,40 \%$ & $62,70 \%$ & $82,20 \%$ & 0,098 \\
\hline Anticoagulante & $69,60 \%$ & $68,80 \%$ & $62,00 \%$ & $63,60 \%$ & 0,828 \\
\hline Antiagregante & $32,60 \%$ & $31,90 \%$ & $31,40 \%$ & $22,70 \%$ & 0,709 \\
\hline \multicolumn{6}{|l|}{ Antidiabéticos } \\
\hline ADOs & $17,00 \%$ & $17,40 \%$ & $9,80 \%$ & $24,40 \%$ & 0,799 \\
\hline Insulina & $10,60 \%$ & $10,90 \%$ & $19,60 \%$ & $13,30 \%$ & \\
\hline ADOs + Insulina & $2,10 \%$ & $2,20 \%$ & $2,00 \%$ & $2,20 \%$ & \\
\hline \multicolumn{6}{|l|}{ Hierro/EPO } \\
\hline Hierro & $23,40 \%$ & $38,30 \%$ & $12,20 \%$ & $11,60 \%$ & 0,029 \\
\hline EPO & $2,10 \%$ & $6,40 \%$ & $2,00 \%$ & $2,30 \%$ & \\
\hline EPO + Hierro & $2,10 \%$ & $2,10 \%$ & $0,00 \%$ & $0,00 \%$ & \\
\hline
\end{tabular}

ADOS: antidiabéticos orales; ARA II: Antagonistas de los Receptores de la Angiotensina II; EPO: Eritropoyetina; EPOC: Enfermedad Pulmonar Obstructiva crónica; FEVI: Fracción de Eyección del Ventrículo Izquierdo; HTA: Hipertensión Arterial; IECAs: Inhibidores de la Enzima Conversora de la Angiotensina; IMC: Índice de Masa Corporal; NT-proBNP: fracción aminoterminal del péptido natriurético cerebral; NYHA: New York Heart Association; PAD: Presión Arterial Diastólica; PAS: Presión Arterial Sistólica.

Tabla 4. Resultados al final del seguimiento según Presión Arterial Sistólica y Presión Arterial Diastólica.

\begin{tabular}{lccccc}
\hline PAS & Q1 (<119,25) & Q2 (119,25-137,5) & Q3 (137,5-157,75) & Q4 (>157,75) & p \\
\hline & $\mathbf{n}=\mathbf{5 5}$ & $\mathbf{n}=\mathbf{5 5}$ & $\mathbf{n}=\mathbf{5 5}$ & $\mathbf{n = 5 5}$ & \\
Mortalidad 10 mes & $1,80 \%$ & $0,00 \%$ & $0,00 \%$ & $0,00 \%$ & 0,392 \\
Mortalidad 30 mes & $11,10 \%$ & $1,80 \%$ & $1,80 \%$ & $1,90 \%$ & 0,031 \\
\hline
\end{tabular}




\begin{tabular}{|c|c|c|c|c|c|}
\hline Mortalidad 60 mes & $10,60 \%$ & $5,60 \%$ & $3,80 \%$ & $1,90 \%$ & 0,251 \\
\hline Mortalidad total & $20,00 \%$ & $9,10 \%$ & $5,50 \%$ & $5,50 \%$ & 0,034 \\
\hline \multirow[t]{2}{*}{ PAD } & Q1 (<64) & Q2 (64-72) & Q3 (72-81) & $\mathrm{Q} 4$ (>81) & $p$ \\
\hline & $n=57$ & $n=56$ & $n=54$ & $n=53$ & \\
\hline Mortalidad 10 mes & $1,80 \%$ & $0,00 \%$ & $0,00 \%$ & $0,00 \%$ & 0,415 \\
\hline Mortalidad 3 mes & $8,90 \%$ & $5,40 \%$ & $1,90 \%$ & $0,00 \%$ & 0,093 \\
\hline Mortalidad 60 mes & $10,00 \%$ & $5,80 \%$ & $3,80 \%$ & $1,90 \%$ & 0,308 \\
\hline Mortalidad total & $17,50 \%$ & $12,50 \%$ & $5,60 \%$ & $3,80 \%$ & 0,059 \\
\hline
\end{tabular}

